\title{
Decision process and preferences over risk under the "endogenous decision rule": Results from a group experiment
}

\author{
Andrea Morone* • Simone Nuzzo • Tiziana Temerario \\ Università degli Studi di Bari Aldo Moro, Italy
}

Received: 3 March 2020

Revised: 29 June 2020

Accepted: 29 July 2020

\begin{abstract}
The recent literature on individual vs. group decision-making, in risky contexts, has brought about divergent results, mainly depending on the institutional rules through which groups take decisions. While some studies where group decisions relied on the majority rule showed no appreciable difference between individuals and groups' preferences, others where unanimity among group members was required found collective decisions to be less risk averse than individual ones. We elicited groups' preferences over risk using what we defined "endogenous decision rule", i.e. leaving groups free to endogenously solve the potential disagreement among their members, just as in many real life situations. Our results unambiguously show that individuals are more risk seeker than groups when facing gambles with positive expected payoff difference and more risk averse in the opposite case.
\end{abstract}

Keywords: individual decision-making; group decision-making

JEL Classification Codes: C9

\section{Introduction and motivational framework}

Although economists have traditionally considered decision makers as isolated individuals, the reality shows us that collective decisions take place in any corner of society. To give few examples, strategic company policies spring from senior managers operating in a group environment (board of directors); public policies in advanced democracies reflect the outcome of an institutional and collective political process; most hiring decisions involve a group of human resource professionals and so on.

The wide recurrence of group decisions in everyday contexts has led many researchers to devote increasing attention to the mechanisms driving collective choices, thus raising the question of whether the groups' outcome results from a simple aggregation of individual preferences or is instead affected by some interaction among group members. Particularly, the driving force underlying this relatively new research strand has been the comprehension of the extent to which a group environment can exert some influence on individuals, thus leading them

\footnotetext{
*Corresponding author. E-mail: a.morone@gmail.com.

Citation: Morone, A., Nuzzo, S., Temerario, T. (2021) Decision process and preferences over risk under the "endogenous decision rule": Results from a group experiment, Economics and Business Letters, 10(2), 108-116.
}

DOI: $10.17811 /$ ebl.10.2.2021.107-115 
to behave differently depending on whether they have to take decisions in isolation rather than collectively. Among the many factors and mechanisms that are in place when the group's members interact, the decision rule is probably the one that has attracted most attention over the last decade. Indeed, plenty of experimental literature (see, for example, Baker at al., 2008; Shupp and Williams, 2008; Masclet et al., 2009; Harrison et al., 2012; Zhang and Casari, 2012; Brunette et al., 2015) has exhaustively provided evidence of the degree at which the group's outcome may be particularly sensitive to the decision rule that the group has follow to come to the final decision. In this respect, the majority and the consensus (or unanimity) rules are the ones on which most experimental studies have been recently focusing on. For example, in Masclet et al. (2009) and Shupp and Williams (2008), unanimity rule leads to the conclusion that groups are more risk-averse. However, in Zhang and Casari (2012) groups appear less riskaverse. Harrison at al. (2012) did not detect any group effect using the majority rule, while Baker et al. (2008) found a more risk-averse group's behavior. Probably, the reasons behind the large recurrence of the two rules in research studies is ingrained in their frequent adoption in real life situations as well as in the wide-ranging merits that each rule carries over. The majority rule $^{1}$ is often the method of choice associated with parliamentary decisions as well as with large public assemblies or small governing boards. As discussed by Gastil (1993), the majority rule advocates the merits of (i) ensuring equal power among group members by depriving them of their veto power; (ii) fostering the rise of different views, especially when speaking turns are alternated between the supporters and opponents of the proposal under scrutiny; (iii) allowing quick decisions. Nevertheless, the majority rule is not exempt from pitfalls. As a first issue, once a well-defined majority is identified, those favouring the prevailing view may not be particularly prone to listen to the minority's arguments, thus impairing the emergence of different opinions and the overall quality of the debate. As a further point, the decisional process under the majority rule may cause a segmentation of the group's membership into two or more factions, with one constituting systematically the majority over a large number of proposals. As a consequence, some members may feel all the time stuck in a permanent minority, and their commitment to contributing constructively to the deliberation process may dramatically decline. Differently, the consensus decision rule relies upon discussion, articulation, attempts to change the minds of group members, and often originates an outcome which consists of a reformulated version of a popular proposal that was not able to gather full agreement among the group's members. If, on one hand, each member has the huge power to unilaterally block a proposal, on the other hand, any abuse of the "blocking privilege" is very likely to awake social pressures and to impair cohesion within the group. When a decision is taken through consensus, the fact that the approval of a proposal remains subjected to full agreement among all members ensures that minorities' points of view are carefully embraced and that opportunities to speak are all the time safeguarded. In addition, the consensus rule stimulates strong commitment toward both the decision process and the decision outcome. While the former is ensured by the awareness of the great power and responsibility that each subject has during the decisional process, the latter arises from the recognition that a given outcome, although not being the best in an absolute sense, it is still probably the best one upon which the group could agree. By contrast, consensus rules often produce slow decisions and, in practice, members can all the time threaten a conflict if there is no agreement with their own view.

The pros and cons of these two conventional rules have been investigated in several experimental settings (a detailed review will be provided in the next paragraph) which, despite being different in some features, have shared the common practice of presenting the decision rule as exogenous. Indeed, in the traditional way of intending the deliberation process, the decision rule has at least two connotations. Firstly, the decision rule is predetermined. This

\footnotetext{
${ }^{1}$ Although the majority rule is, by default, thought of as simple majority (more than $50 \%$ of votes), it may also be based on any fraction of votes greater than one half and less than the total number of the group's members.
} 
means that the rule predates the formation of the group. Secondly, the decision rule is exogenous. This signifies that the rule has an external source of determination, which typically coincides with the group's by-laws. Therefore, traditional experimental studies have exogenously manipulated the decision rules to shed light on the effects that different rules produce on the group's outcome.

Contrarily to this view, in comparing individual and group's decision making over a set of risky choices, our research proposes a "reconceptualization" of the decision rule, which materialises in the absolute novelty of "internalizing" the decision rule. Indeed, in our study, group members are (i) free to discuss without any restriction of time prior to coming to the final decision, and (ii) free to choose any method to overcome potential disagreement among group members. Since the group's final deliberation is not conveyed by any exogenously imposed breaking rule, we namely refer to our mechanism with the terminology "endogenous decision rule" (EDR). The reconceptualization which we propose finds its raison d'etre in at least three aspects, which have a factual, democratic, and technical fashion respectively. As a first point, Putnam and Stohl (1990) assert that researchers ought to face the evidence that outside the laboratory there are plenty of small groups which have different historical origins and share different habits. Typically, such groups are informal, newly formed, and meet spontaneously to take decisions. Most of the times, within these groups, there is no formal adoption of any decision rule and the final decision is not preceded by official votes. In addition to this, the recent development of social technologies has driven some political parties to give their followers the possibility to actively contribute to internal decisions as well as to propose their view on a wide range of political issues. The second aspect constituting the foundations of our reconceptualization has a socio-democratic connotation. The basic idea is that it may be particularly difficult to frame democratic decisions in deliberative procedures that have been set out by entities outside the group. As reported by Gastil (1993), people who serve on a jury in a criminal case may perceive the decisional rule they must use as reasonable just because such rule is the outcome of a legitimate process which may have political rather than historical roots (i.e. it is contemplated in their constitutional paper). It is easy to recognize that an experimental study does not work that way and that the subjects gathered in a laboratory lack the precedent of framing the exogenous decision rule in a legitimate source. For this reason, the experimental investigation of any deliberation process should allow the groups to select their own decision rules endogenously. As a final point, a technical drawback in setting an exogenous default rule is that of inducing the subjects to behave according to such rule. In this sense, Baker et al. (2008) reported that $94.7 \%$ of groups decisions turned out to be consistent with the majority rule just because that rule would have been adopted in case of disagreement. Therefore, the adoption of the endogenous decision rule would eliminate this bias.

\section{Experimental design and procedure}

The experiment was paper and pencil and conducted at the ESSE laboratory of experimental economics at the University of Bari Aldo Moro, where a sample of 300 students was randomly selected from different undergraduate courses. The $48 \%$ of our sample was composed by male, and the average age was 21 . Each participant had to attend two separate parts (i.e. individual-part and group-part). On each of the two parts the subjects were presented the same 10 pairwise choice lotteries (see the first two panel of Table 1).

The lotteries were presented as segmented circles (see Figure 1).

The individual-part lasted between 5 and 10 minutes; the group-part lasted between 10 and 30 minutes. The time varied not only between the single parts but also among subjects since they were explicitly encouraged to proceed at their own pace. 
Table 1. The ten-paired lottery-choice decisions under risk. Panel 3 from the left reports the expected value (EV) of each lottery, and the expected payoff difference (EPD) between lottery A and lottery B.

\begin{tabular}{|c|c|c|c|c|c|c|c|c|c|c|c|}
\hline \multicolumn{4}{|c|}{ Lottery A } & \multicolumn{4}{|c|}{ Lottery B } & \multirow{2}{*}{$\mathrm{EV}(\mathrm{A})$} & \multirow{2}{*}{$\mathrm{EV}(\mathrm{B})$} & \multirow{2}{*}{ EPD } & \multirow{2}{*}{$\begin{array}{c}\text { Open CRRA interval if } \\
\text { subject switches to } \\
\text { lottery B }\end{array}$} \\
\hline Prob. & Gain & Prob. & Gain & Prob. & Gain & Prob. & Gain & & & & \\
\hline 0.1 & 50 & 0.9 & 40 & 0.1 & 96.25 & 0.9 & 2.5 & 41 & 11.88 & 29.12 & $-\infty,-1.71$ \\
\hline 0.2 & 50 & 0.8 & 40 & 0.2 & 96.25 & 0.8 & 2.5 & 42 & 21.25 & 20.75 & $-1.71,-0.95$ \\
\hline 0.3 & 50 & 0.7 & 40 & 0.3 & 96.25 & 0.7 & 2.5 & 43 & 30.62 & 12.38 & $-0.95,-0.49$ \\
\hline 0.4 & 50 & 0.6 & 40 & 0.4 & 96.25 & 0.6 & 2.5 & 44 & 40.00 & 4.00 & $-0.49,-0.15$ \\
\hline 0.5 & 50 & 0.5 & 40 & 0.5 & 96.25 & 0.5 & 2.5 & 45 & 49.37 & -4.37 & $-0.15,0.15$ \\
\hline 0.6 & 50 & 0.4 & 40 & 0.6 & 96.25 & 0.4 & 2.5 & 46 & 58.75 & -12.75 & $0.15,0.41$ \\
\hline 0.7 & 50 & 0.3 & 40 & 0.7 & 96.25 & 0.3 & 2.5 & 47 & 68.12 & -21.12 & $0.41,0.68$ \\
\hline 0.8 & 50 & 0.2 & 40 & 0.8 & 96.25 & 0.2 & 2.5 & 48 & 77.5 & -29.5 & $0.68,0.97$ \\
\hline 0.9 & 50 & 0.1 & 40 & 0.9 & 96.25 & 0.1 & 2.5 & 49 & 86.87 & -37.87 & $0.97,1.37$ \\
\hline 1.0 & 50 & 0.0 & 40 & 1.0 & 96.25 & 0.0 & 2.5 & 50 & 96.25 & -46.25 & $1.37, \infty$ \\
\hline
\end{tabular}

Figure 1. The Presentation of Lotteries.

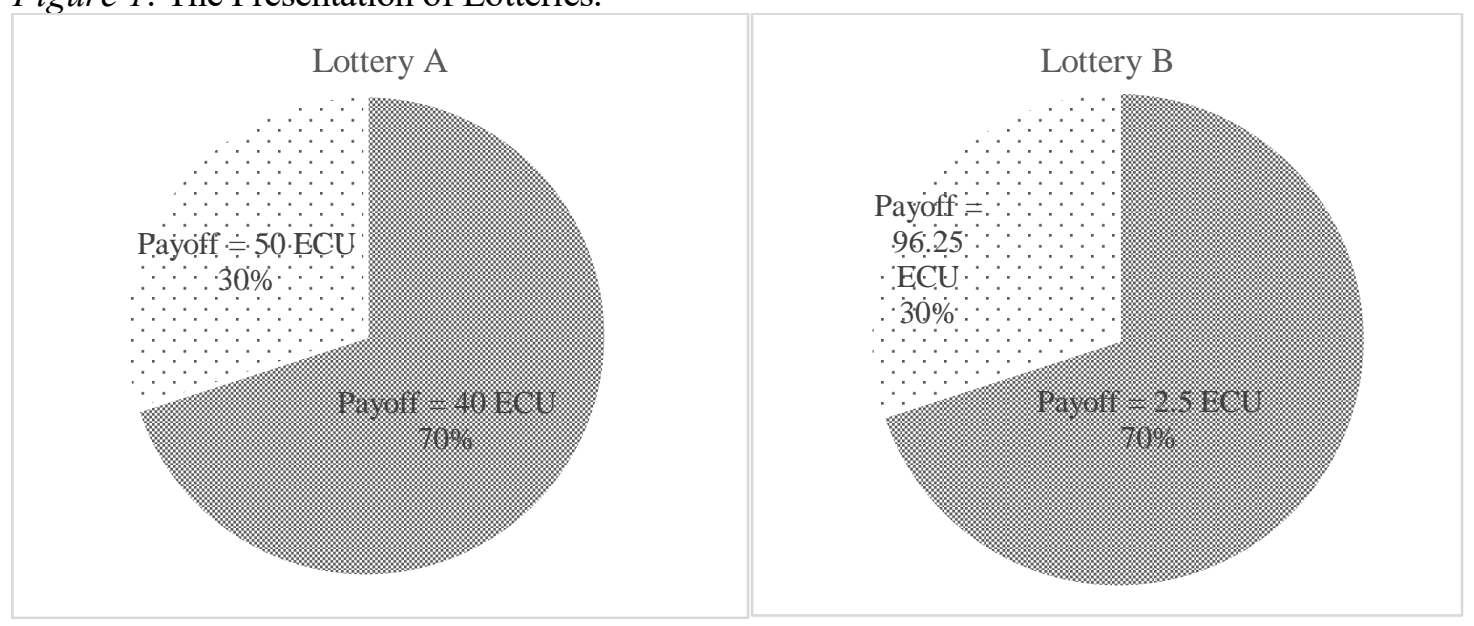

Each individual had to choose one lottery and take note on a form handed out previously. In the group part, subjects were randomly allotted to groups of three persons each and faced the same 10 pairwise choices as in the individual part. Group composition remained fixed over the ten decision problems. Again, each group was provided with a form in which the final group decision had to be reported. The members within each group could communicate face-to-face ${ }^{2}$ without any limits of time, since the payoff for each decision was bounded between $2.5 \mathrm{ECU}$ and 96.25 ECU. The longer subjects had taken to reach an agreement in the group the smaller would have been their payment per hour; this gave them a strong incentive not to discuss forever. Baker et al. (2008) allowed a "cheap talk" among members but participants were aware that, if an agreement was not reached, a default rule (majority) would have been applied. Instead, in our experiment group members were totally free to talk ${ }^{3}$ as well as to adopt any internal method to reach a decision.

Once a decision was taken, subjects moved to the next decision problem. Groups were sufficiently apart so that each group could not listen to others' discussions.

After a subject had completed all two parts one question of one part was selected randomly and played out for real. The average payment to the subjects was approximately $€ 20$.

\footnotetext{
${ }^{2}$ While in some papers group members usually have discussion via computer (Masclet et al., 2009; Zhang and Casari, 2012; Brunette et al., 2015), O'Neill et al. (2016) showed that face-to-face teams were more effective on all decision behaviours

${ }^{3}$ They have to report the group decision, but they are free to take the decision unanimously or by majority or following other rules. The decision rule was not exogenously imposed but emerged in the group. No record of their conversation was taken.
} 


\section{Analysis and results}

Prior to introduce our core results, it is useful to recall that a rational decision maker with monotonic preferences is expected to switch from the safer to the riskier option just once and never switch back. Like in many other experiments (see, for example, Keck et al. 2014), we observed cases of multiple switching. Indeed, some subjects (groups) switched from A to B and vice versa more than once, showing such a kind of inconsistency or indifference over a certain range. In detail, multiple switching occurred in 24 out of 300 instances in the individual part, and in 5 out of 100 cases in the group part. A two-sample proportion test shows that the multiple switching frequency is not different between the individual and group part ( $m=300, n=100$, $\mathrm{z}=1.00, p$-value $=0.3164$ ).

In order to estimate subjects' risk attitude, we resort to the conventional constant relative risk aversion (CRRA) function

$$
U(y)=\frac{y^{1-r}}{1-r}
$$

where, $r$ is the CRRA coefficient. If $r=0$ the subject is risk neutral, if $r>0$ he is risk averse, if $r<0$ he is risk loving. We preliminary compared the $r$ distributions from the individual and group part running a non-parametric Mann-Whitney U test. Our results show that this test cannot reject the null hypothesis of equality between the distributions of the $r$ coefficients in the two parts $(m=95, n=276, z=0.098, p \text {-value }=0.9223)^{4}$. Then, we provide evidence that individuals' risk attitude were not different from that of groups.

Figure 2 shows the percentage of safe option (A) choices along with the ten decision problems in the two tasks. In theory, a risk-neutral subject is expected to switch from A to B when the expected payoff of the two lotteries is equal, i.e. between the $4^{\text {th }}$ and $5^{\text {th }}$ decision problem. While a switch in later decisions reveals risk aversion, a switch in earlier decisions reveals risk-seeking behaviour.

Figure 2. Fraction of individuals and groups who chose the safe option A over the ten decision problems.

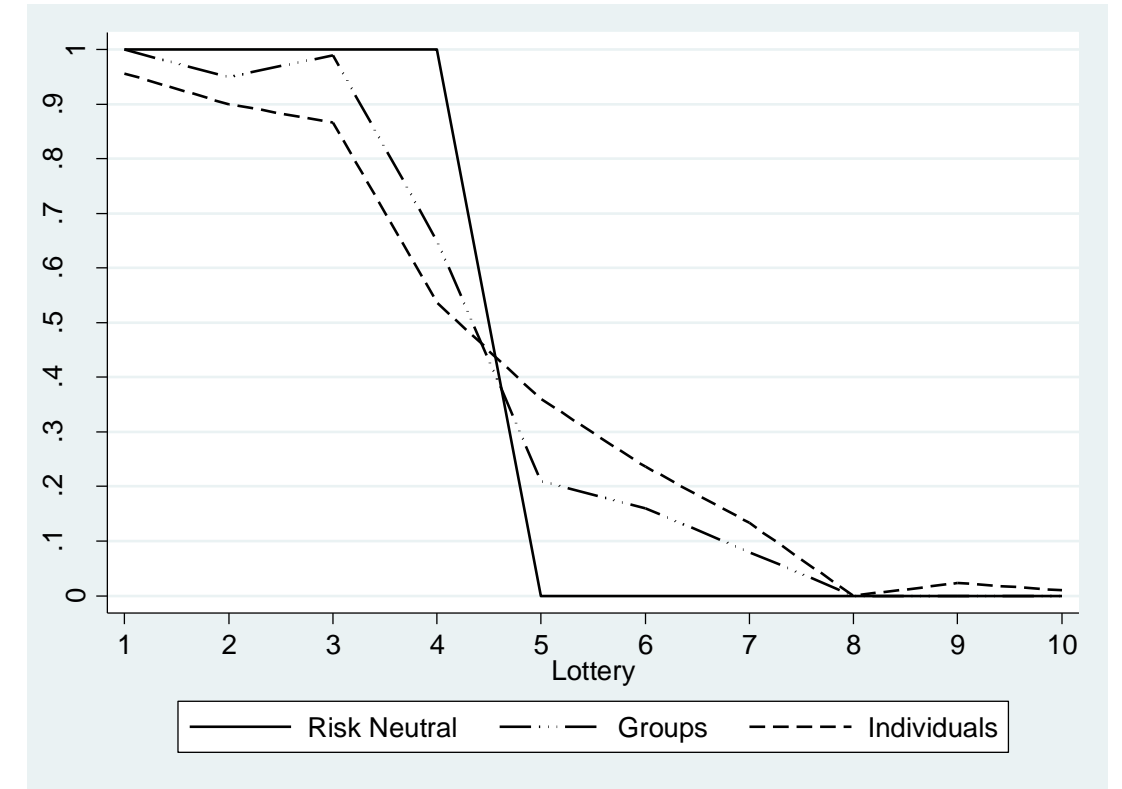

\footnotetext{
${ }^{4}$ According to Jacobson and Petrie (2009) we removed multiple switchers from the analysis, since the analysis with all the observations included (as suggested by Holt and Laury, 2000) provides the same results. This analysis is available on request.
} 
Table 2. Probability of choosing the safe option (A): Logit model.

\begin{tabular}{lcccc}
\hline Dep. Var. Pr(A_choice) & 1_10 Choice & 1_4 Choice & 5_10 Choice & $\begin{array}{c}\text { Intecartion } \\
\text { Model }\end{array}$ \\
\hline \hline Group & 0.0145 & $0.690^{* * *}$ & $-0.589^{* * * *}$ & $0.690^{* * * *}$ \\
& $(0.0961)$ & $(0.181)$ & $(0.171)$ & $(0.181)$ \\
Neg_epd_choices & $-3.702^{* * *}$ & $/ /$ & $/ /$ & $-3.435^{* * *}$ \\
& $(0.0943)$ & $/ /$ & $/ /$ & $(0.104)$ \\
Constant & $1.765^{* * *}$ & $1.642^{* * *}$ & $-1.822^{* * *}$ & $/ /$ \\
& $(0.159)$ & $(0.228)$ & $(0.199)$ & $/ /$ \\
GroupXneg_epd_choices & $/ /$ & $/ /$ & $/ /$ & $-1.279 * * *$ \\
& $/ /$ & $/ /$ & $/ /$ & $(0.248)$ \\
Session Control & Yes & Yes & Yes & Yes \\
Observations & 4,000 & 1,600 & 2,400 & 4,000 \\
Pseudo_r2 & 0.4220 & 0.0172 & 0.0148 & 0.4274 \\
Prob > chi2 & 0.0000 & 0.0092 & 0.0062 & 0.0000 \\
\hline \hline
\end{tabular}

Note: "1 10 Choice" denotes the entire set of decision problems. "1 4 Choice" refers to the restricted model in which only the decision problems from the $1^{\text {st }}$ to the $4^{\text {th }}$ have been accounted for. "5 10 Choice" refers to the restricted model in which we only include the decision problems from the $5^{\text {th }}$ to the $10^{\text {th }}$. In the last column from the left we report the results from the same model including the interaction between "Group" and "Neg_epd_choices". Heteroskedasticity-consistent standard errors (robust) in parentheses. $* * * \mathrm{p}<0.01, * * \mathrm{p}<0.05, * \mathrm{p}<0.1$.

We employ the following Logit model to explain the determinants of the likelihood to choose the safe option (A).

$$
y_{i}=\alpha+\beta_{1} \cdot \text { Group }_{i}+\beta_{2} \cdot \text { Neg_epd_choices }_{i}+\sum_{j=1}^{k} \gamma_{j} \cdot \text { Session }_{j}+\varepsilon_{i}
$$

Where $y_{i}$ is a dichotomous dependent variable which takes on value 1 if the decision maker $i$ chose the safe option (A) and 0 otherwise. $\operatorname{Group}_{i}$ is a dummy variable which assumes value 1 if the decision maker $i$ is a group and 0 if the choice is instead taken individually. $N_{e} g_{-} e p d \_c h o i c e s_{i}$ is a dummy which takes on value 1 within the decision problems with a negative expected payoff difference $(E P[A]<E P[B])$, i.e. in all the choices from 5 up to 10 , and 0 otherwise. Finally, $\sum_{j=1}^{k} \gamma_{j} \cdot$ Session $_{j}$ is a vector of dummies which controls for specific session effects. The results from the logit model are summarized in table 2.

Considering the whole set of decision problems, these results show that moving from individual to group decision making does not affect the probability of choosing the safe lottery (A). Ceteris paribus, a switch from positive expected payoff difference (EPD) lotteries (problems from 1 up to 4) to negative EPD lotteries (problems from 5_10) significantly reduces the probability of choosing the safe option (A). This latter result is in line with Baker et al. (2008) and Brunette et al. (2015). Instead, the most interesting achievements come from the restricted model. Indeed, considering only those lotteries with positive EPD, playing in group significantly increases the probability of choosing the option A, thus meaning that individuals are more risk seeking than groups. The opposite pattern is instead interestingly (and significantly) detected in the lotteries with negative EPD, in which individuals appreciably exhibit a greater degree of risk aversion than groups. To better appreciate this trend we augment our model with the interaction term " $\beta_{3}$. Group_X_Neg_epd_choices", and report the output in table 2 and figure 3. 
Figure 3. Adjusted predictions of the interaction "Group_X_Neg_epd_choices" on the probability of choosing the safe option (A).

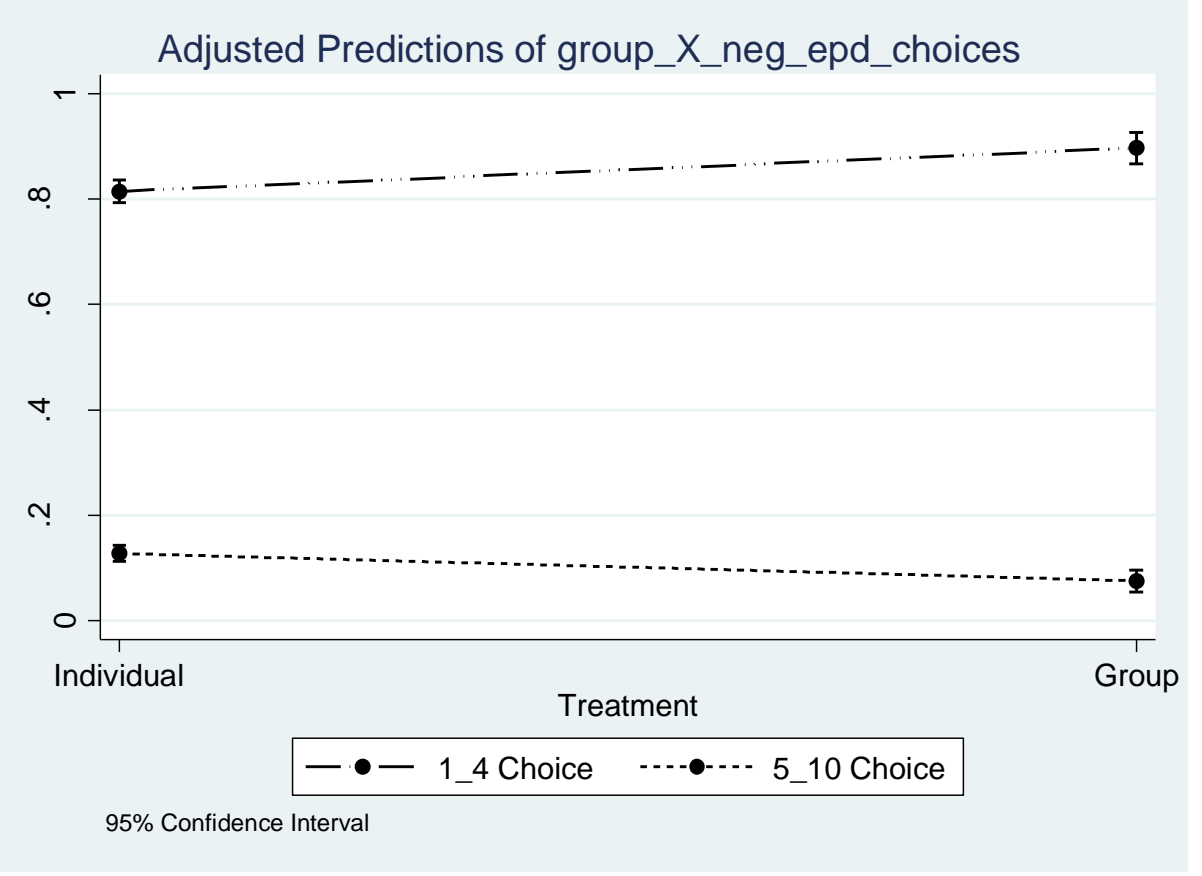

We find a negative and significant interaction between the variable "Group" and "Neg_epd_choices", which highlights how the effect of playing in group - rather than individually - on the average predicted probability of choosing the safe option (A) crucially changes depending on whether the positive rather than negative domain of the EPD between the two gambles is considered.

\section{Conclusions}

This paper investigates whether subjects' attitude toward risk changes depending on whether decisions are taken individually or in a group. We did not impose an exogenous disagreementbreaking rule, but we left each group free to resolve the disagreement. The only restriction we imposed was that subjects had to come to a group decision. However, they were totally free to adopt any method to come to a decision and talk as much as they needed. We clearly show that, although no appreciable difference in risk attitudes arises comparing individual and group behaviour over the whole set of decision problems, groups always better proxy the risk neutral behaviour than individuals. In particular, we find that groups are more likely than individuals to choose the safe option when playing lotteries with positive expected payoff difference and less likely than individuals to choose the safe option when playing lotteries with negative expected payoff difference. In both the domains, groups' decisions are closer to risk neutrality than individuals' decisions. This may shed a light to a sort of rational "polarization" of group behaviour with respect to individuals. Moreover, reading these results with regard to the previous literature, we speculate that groups may have applied unanimity rule in decisions 1 to 4 and majority rule in the decisions 5 to 10 . However, this conjecture needs to be verified with dedicated laboratory experimental designs.

\section{References}

Baker, R. J., Laury, S. K., and Williams, A. W. (2008) Comparing Small-Group and Individual Behavior in Lottery-Choice Experiments, Southern Economic Journal, 75(2), 367-382.

Brunette, M., Cabantous, L., and Couture, S. (2015) Are individuals more risk and ambiguity 
averse in a group environment or alone? Results from an experimental study, Theory and Decision, 78(3), 357-376.

Fischbacher, U. (2007) z-Tree: Zurich toolbox for ready-made economic experiments, Experimental economics, 10(2), 171-178.

Gastil, J. (1993) Democracy in small groups: Participation, decision making and communication, Philadelphia PA: NewSociety.

Harrison, G. W., Morten, L., Rutström, E., and Tarazona-Gomez, M. (2012) Preferences over Social Risk, Oxford Economic Papers, 65(1), 25-46.

Holt, C. A., and Laury, S. K. (2002) Risk Aversion and Incentive Effects, American Economic Review, 92(5), 1644-1655.

Jacobson, S., and Petri, R. (2009) Learning from mistakes: What do inconsistent choices over risk tell us?, Journal of Risk and Uncertainty, 38(2), 143-158.

Masclet, D., Loheac, Y., Denant-Boemont, L., and Colombier, N. (2009) Group and Individual Risk Preferences: a Lottery-Choice Experiment, Journal of Economic Behavior and Organization, 70(3), 470-484.

Shupp, R. S., and Williams, A. W. (2008) Risk preference differentials of small groups and individuals, The Economic Journal, 118(525), 258-283.

Zhang, J., and Casari, M. (2012) How Groups Reach Agreement, in Risky Choices: An Experiment, Economic Inquiry, 50(2), 502-515. 


\section{Additional material - Experiment instructions (translated from the original in Italian)}

\section{Welcome to the Experiment}

This is an experiment to investigate your evaluation of lotteries. At the end of the experiment you will receive a payoff based on the decisions you make according to the rules set out at the end of the experiment. Please read the instructions very carefully before starting the experiment. In particular, make sure that you have understood the payoff procedure before starting.

You will face 10 pairs of lotteries. For each pair you will be asked to choose the lottery that you prefer the most. Below is an example of the pairs of lotteries you can face:

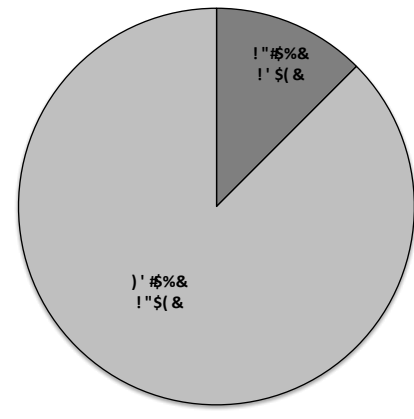

Lottery A

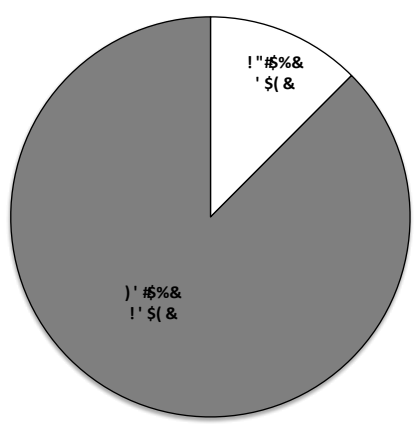

Lottery B

You will have to choose between Lottery A and Lottery B. Once you complete this task, one out of the 10 pairs of lotteries will be randomly chosen. The lottery that you stated as the most preferred will then be played for real. 